\title{
Energy Management Systems for Optimal Operation of Electrical Micro/Nanogrids
}

\author{
Maria Carmela Di Piazza (D) \\ Consiglio Nazionale delle Ricerche (CNR), Istituto di Ingegneria del Mare (INM), via Ugo La Malfa 153, \\ 90146 Palermo, Italy; mariacarmela.dipiazza@cnr.it
}

check for updates

Citation: Di Piazza, M.C. Energy Management Systems for Optimal Operation of Electrical

Micro/Nanogrids. Energies 2021, 14, 8469. https://doi.org/10.3390/ en14248469

Received: 29 November 2021 Accepted: 3 December 2021 Published: 15 December 2021

Publisher's Note: MDPI stays neutral with regard to jurisdictional claims in published maps and institutional affiliations.

Copyright: (c) 2021 by the author. Licensee MDPI, Basel, Switzerland. This article is an open access article distributed under the terms and conditions of the Creative Commons Attribution (CC BY) license (https:/ / creativecommons.org/licenses/by/ $4.0 /)$.

\section{Introduction}

Energy management systems (EMSs) have been introduced in electrical power systems to optimize operations of the electrical grid infrastructure and to provide support to the grid operator in terms of optimized decisions. Through their key functions of monitoring, control, optimization of flows, and use of electrical power, EMSs allow customers to play an active role in the energy market, as well.

According to a general classification, the main objectives of an EMS can be divided into diverse categories, such as energy efficiency improvements, demand profile shaping, utility and cost optimization, price stabilization, and pollutant emission reduction. Moreover, plenty of methods and tools for pursuing management objectives are currently available, ranging from classical and meta-heuristic optimization methods to artificial intelligence and machine learning-based techniques.

In the case of electrical micro/nanogrids, the development of suitable EMSs is even more crucial, due to need to correctly handle the uncertainties and intermittency of renewables and the uncertainty of the power demand, while maintaining a stable operation. On such a basis, EMSs are nowadays considered one of the most relevant technical solutions for enhancing the efficiency, reliability, and economy of smart micro/nanogrids, both in terrestrial and vehicular applications. For this reason, the recent technical literature includes numerous contributions on EMSs for residential, commercial, and vehicular micro/nanogrids that encompass renewable generators, different typologies of electrical loads, and energy storage systems (ESSs) usually relying on batteries.

Despite the considerable interest of the scientific community and industrial stakeholders on the development of effective and reliable EMSs, the EMSs proposed so far were not always conceived with respect to fostering their widespread and rapid adoption. For this to occur, several issues remain to be tackled. First, EMSs should be seamlessly integrated with the ecosystem of micro/nanogrid components; secondly, EMSs should interfere as little as possible with the comfort and habits of electricity market customers. Furthermore, the energy management algorithms should preferably provide simultaneous advantages for both the end-user and the grid operator.

On account of the previous considerations, the goal of this Special Issue is to bring together technical and scientific contributions addressing topical issues related to the development of EMSs specially intended for the optimal operation of electrical micro/nanogrids.

As Guest Editor of this Special Issue, I was able to ascertain the benefits of expanding the knowledge on EMSs in the field of electrical micro/nanogrids; moreover I was able to observe the relevance of several topics related to the considered technical field, and covered by the submitted papers, for example: the optimization of electrical power flows, the development of EMSs for smart buildings/smart homes, the development of EMSs based on electric vehicle integration in the microgrid, the development of suitable models of batteries, the suitable use of forecasting methodologies within an EMS, and the optimal use of ESSs in a microgrid. 
The positive response from the scientific community to the proposed Special Issue was reflected in the variety and high-quality of the submitted contributions and demonstrated the significance of the subject together with the need to keep alive the scientific debate on such a topical issue.

In the following section, a brief review of the papers published in this Special Issue, roughly classified according to the main identified thematic areas, is provided to help the reader to acquire knowledge and take advantage of the original scientific results provided in this volume.

\section{Short Review of the Contributions in This Special Issue}

This Special Issue includes seven articles authored by international research teams from several countries. For a straightforward browsing of the volume content, the articles can be considered grouped into the following themes:

- EMSs for smart buildings/homes.

- Control and management of microgrids.

- ESSs modeling and identification.

- Forecasting in EMSs.

As far as the first theme is concerned, Elkazaz et al. in [1] present an exhaustive study assessing the performance of a home energy management system (HEMS) conceived for minimizing daily energy costs, reducing energy lost to the utility, and improving photovoltaic (PV) self-consumption by controlling a suitable battery storage system (BSS). The article investigates the influence of several factors, such as the forecasting errors, the overnight charging level of the battery, the sample time of the control, the battery capacity, and the tariff policy. To carry out the proposed analysis, data for energy consumption and generation collected from real prosumers across the UK have been used.

In the article by Simmini et al. [2], a model predictive control (MPC)-based technique is proposed for the efficient management of a home nanogrid equipped with a PV source, an ESS, electric vehicles (EVs), and a connection to the upstream power grid. By exploiting predictions of generation profiles, energy prices, times of departure and arrival of the EV, and daily EV battery energy consumption, the predictive control effectively manages the prosumers' energy resources. The proposed MPC, in particular, fulfils the objectives of minimizing the economic costs of the system and fully charging the EV battery during nighttime, while respecting the maximum exchanged power constraints at the point of connection with the grid.

The control and management of microgrids is dealt with in three articles focused, respectively, on power flow control, on BSSs capability of managing black start and islanding events, and on use of EV fleets as a flexible load for balancing purpose in the microgrid management system [3-5].

Abedini et al. [3] propose an optimal power flow control method for microgrids together with its real-time validation considering a benchmark low-voltage distribution network with distributed energy resources. The control is designed considering a cost function aimed at optimizing various operation indicators, such as distribution losses, current stresses on feeders, and voltage deviations. The article demonstrates that an enhanced operation of the microgrid is attained; specifically, the proposed control allows dynamic and accurate power flow control, enabling the provision of ancillary services to the upstream grid.

Marchgraber and Gawlik in [4] analyze the black-starting and islanding capabilities of a BESS, acting as grid-forming unit, to provide a possible backup supply for a microgrid. The article proposes a master-slave approach, according to which the power infeed of variable renewable energy during island operation of a microgrid is possible, without the need for additional communication. Moreover, the black-start and island operation of a microgrid, under the proposed master-slave approach, is investigated. Both simulations and experimental results are provided. 
Finally, Gruosso and Ruiz in [5] explore the possibility of using EV fleets as a balancing instrument in a microgrid by exploiting their charging operations. In this article, an optimal control problem is formulated where a degree of flexibility is introduced in the recharging process by increasing the interval available to achieve the target $\mathrm{SoC}$ status, at the cost of reducing the time available for the car-sharing service. The cost function allows finding a trade-off between flexibility exploitation to reduce energy costs and the loss in the car-sharing service caused by a longer recharging time. The results show that the EMS can reduce the peak power consumption of the microgrid, by a suitable extension of the charging time.

The role of energy storage in EMSs is pivotal since the ESSs' buffering capability allows scheduling and management of the electrical power flows in a microgrid according to desired objectives. From this standpoint, the modeling and identification of storage systems and devices is a topic of interest in the considered technical field.

In [6], Ospina Agudelo et al. investigate battery impedance modelling issues. In particular, their article introduces three main approaches for the implementation of the time-domain response of battery fractional order models (FOM)s, i.e., multiple RC $(\mathrm{mRC})$, Oustaloup (OU), and Grünwald-Letnikov. A comparison of such approaches in terms of accuracy, computational requirements, and suitability for the time-domain identification of battery impedance is provided. The obtained results put in evidence that the best FOM implementation method depends on the application requirements, where the $\mathrm{mRC}$ approach offers the best accuracy-complexity compromise, which is desirable for real-time simulations oriented to the validation of energy management algorithms.

To conclude, with reference to the theme of forecasting in EMSs, in [7] La Tona et al. provide a contribution for defining the selection criterion of the daily forecasting frequency, in the framework of a rolling-horizon energy management algorithm. In detail, the article analyzes the performance of a sample EMS (reducing the power demand uncertainty in a microgrid), where the forecasting task is committed to a nonlinear autoregressive network with exogenous inputs (NARX) and an artificial neural network (ANN)" with "with exogenous inputs (NARX) artificial neural network (ANN), and is executed according to different daily frequencies. The obtained results reveal a representative trend relating the forecasting execution frequency in the EMS and the reduction of uncertainty in the electrical demand.

Funding: This research received no external funding.

Acknowledgments: The author is grateful to the MDPI Publisher for the invitation to act as Guest Editor of this Special Issue. As Guest Editor, she would like to express her deep appreciation to all authors and reviewers for their efforts to make this Special Issue a success. Moreover, she is grateful to the editorial staff of Energies, especially to Shary Song, for kind co-operation, support, and committed engagement.

Conflicts of Interest: The author declares no conflict of interest.

\section{References}

1. Elkazaz, M.; Sumner, M.; Pholboon, S.; Davies, R.; Thomas, D. Performance Assessment of an Energy Management System for a Home Microgrid with PV Generation. Energies 2020, 13, 3436. [CrossRef]

2. Simmini, F.; Caldognetto, T.; Bruschetta, M.; Mion, E.; Carli, R. Model Predictive Control for Efficient Management of Energy Resources in Smart Buildings. Energies 2021, 14, 5592. [CrossRef]

3. Abedini, H.; Caldognetto, T.; Mattavelli, P.; Tenti, P. Real-Time Validation of Power Flow Control Method for Enhanced Operation of Microgrids. Energies 2020, 13, 5959. [CrossRef]

4. Marchgraber, J.; Gawlik, W. Investigation of Black-Starting and Islanding Capabilities of a Battery Energy Storage System Supplying a Microgrid Consisting of Wind Turbines, Impedance- and Motor-Loads. Energies 2020, 13, 5170. [CrossRef]

5. Gruosso, G.; Ruiz, F.O. Electric Vehicle Fleets as Balancing Instrument in Micro-Grids. Energies 2021, 14, 7616. [CrossRef]

6. Ospina Agudelo, B.; Zamboni, W.; Monmasson, E. A Comparison of Time-Domain Implementation Methods for Fractional-Order Battery Impedance Models. Energies 2021, 14, 4415. [CrossRef]

7. La Tona, G.; Di Piazza, M.C.; Luna, M. Effect of Daily Forecasting Frequency on Rolling-Horizon-Based EMS Reducing Electrical Demand Uncertainty in Microgrids. Energies 2021, 14, 1598. [CrossRef] 